\title{
Characterization of CMS High Granularity Calorimeter Silicon
}

Sensors

Michael Campanella, Florida State University - SULI

Maral Alyari, FermiLab

Ron Lipton, FermiLab

\section{Challenges for detector design at}

\section{High HL - LHC}

- Two major challenges for detectors:

1. Unprecedented radiation dosage:

- Doses up to 2 MGy

- Mitigated by radiation hard detector materials and readout

2. High Pile-up:
- Up to 200 pile-up per event
- Mitigated by high granularity detectors and precise timing

\section{High Granularity Calorimeter (HGCal)}

\section{- Endcaps cannot} withstand HL-LHC conditions

- Must be replaced by HGCal

- Sampling calorimeter

- $600 \mathrm{~m}^{2}$ of Silicon ( 6 million channels)

- $500 \mathrm{~m}^{2}$ of Scintillator ( 400 thousand channels)

Electromagnetic section
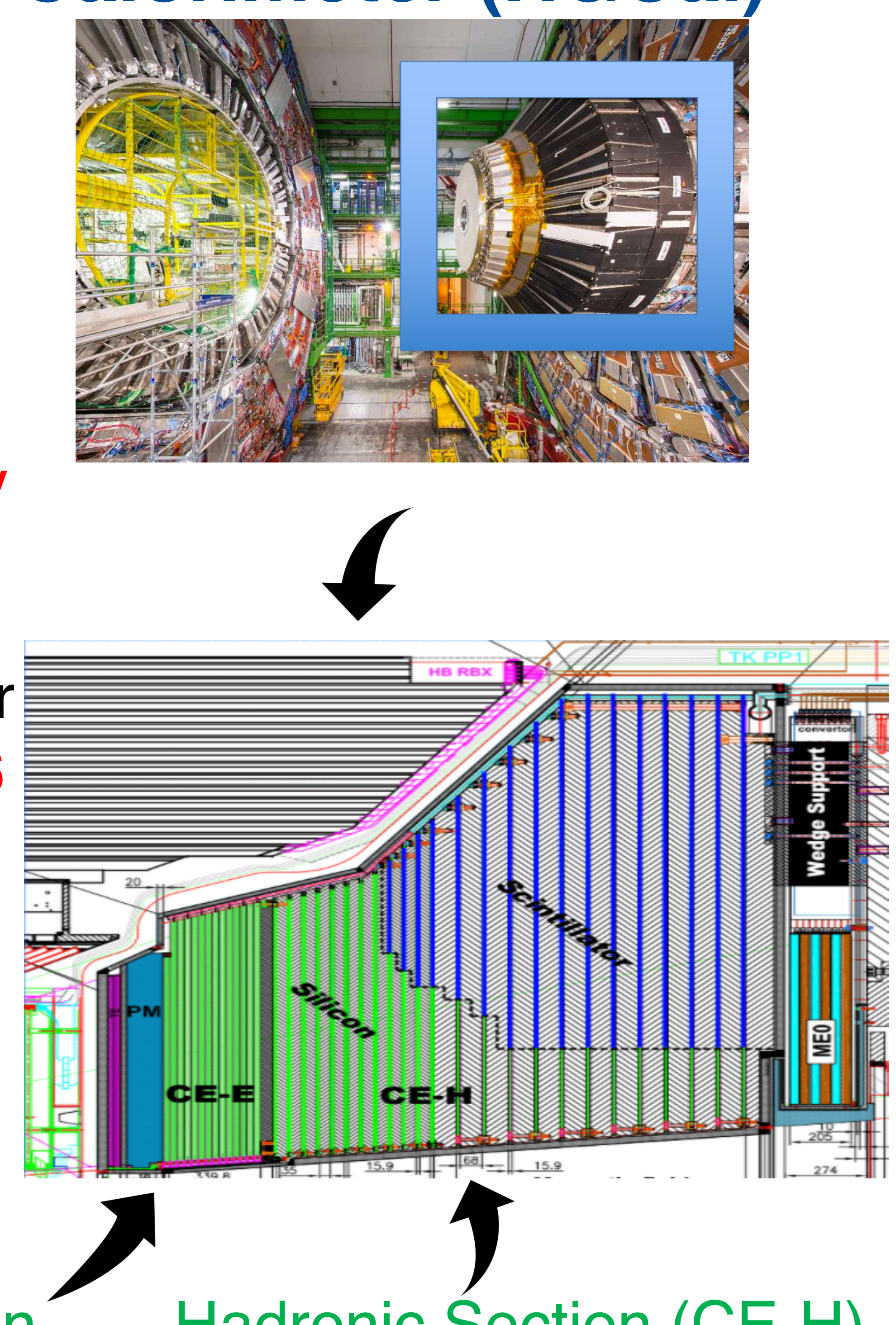

(CE-E)

- 28 sampling layers

- Active elements: Silicon

- Absorber: Lead, CopperTungstate, Copper

Hadronic Section (CE-H)

- 22 sampling layers

- Active elements: silicon (Layers 18) and silicon and scintillator (Layers 9-22)

- Absorber: stainless steel

\section{HGCal Silicon Sensors}

- 8-inches in diameter and hexagonal
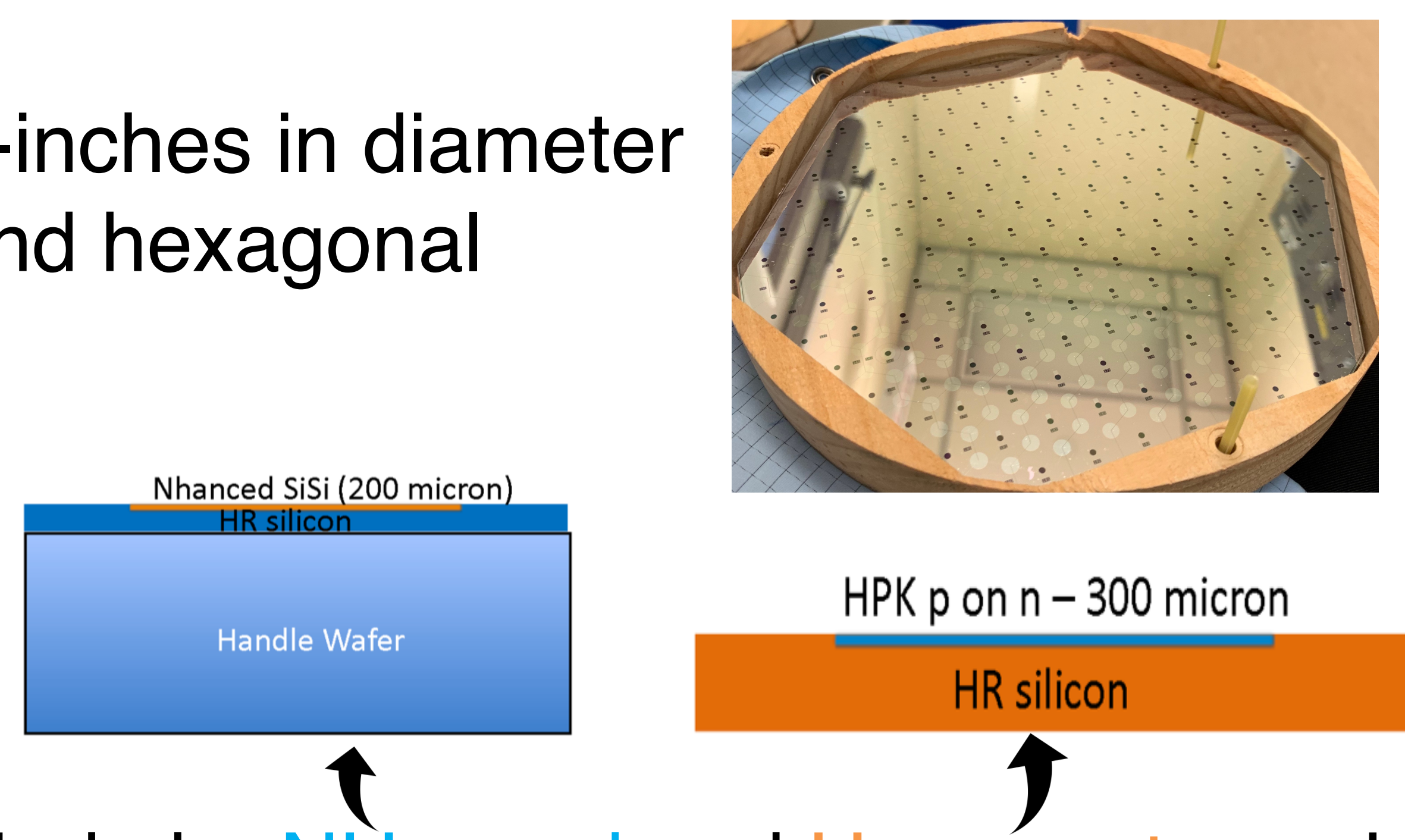

- Made by NHanced and Hamamatsu using different manufacturing techniques
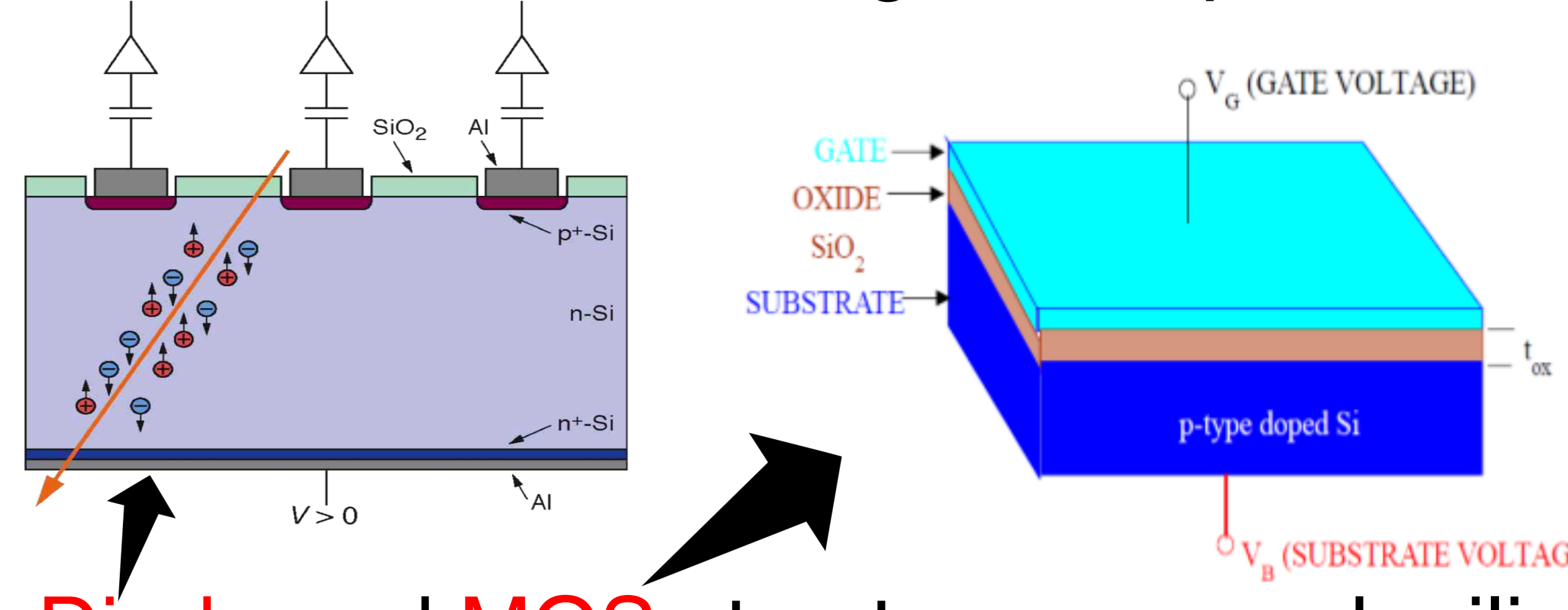

- Diode and MOS structures on each silicon sensor test structures

- This project studies the capacitance of the MOS and diode structures as a function of voltage in order to study the quality of the sensors made by these companies.
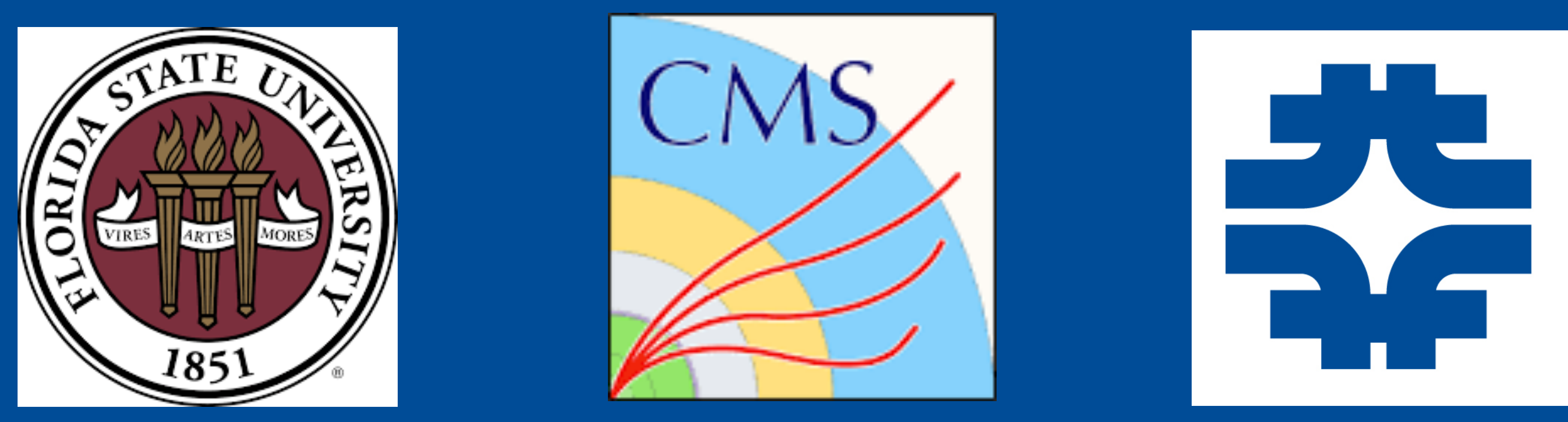

\section{Experimental Set Up}

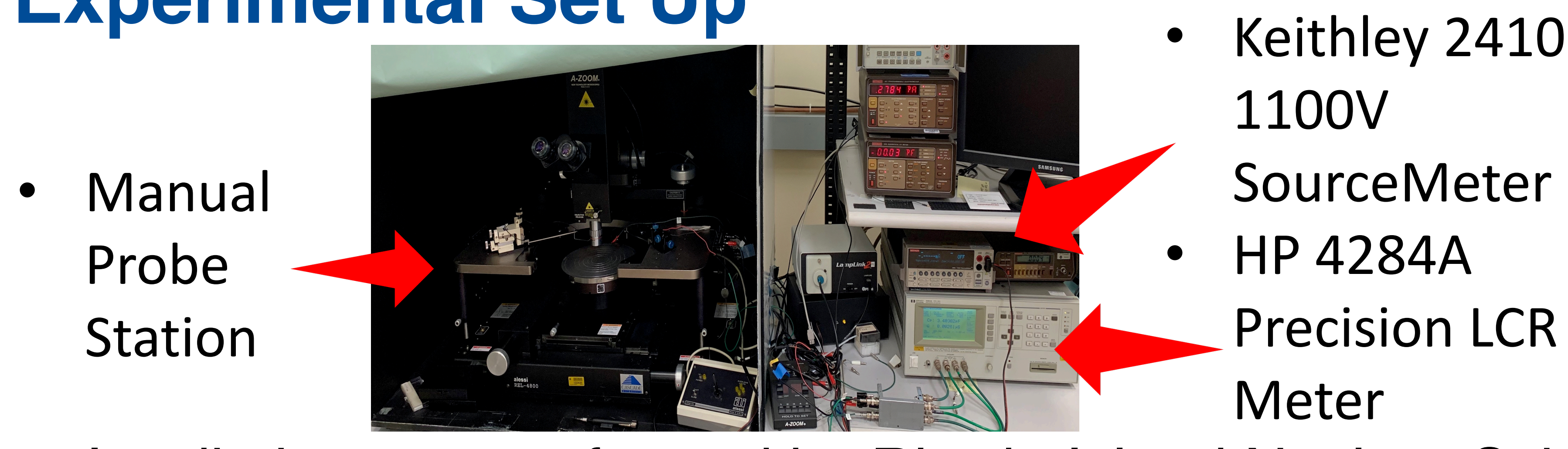

- Irradiation was preformed by Rhode Island Nuclear Science Center with a fluence of $7.5 \times 10^{14} \mathrm{~N}_{\mathrm{eq}}$. $1 / C^{2}-V$ curves of Diodes

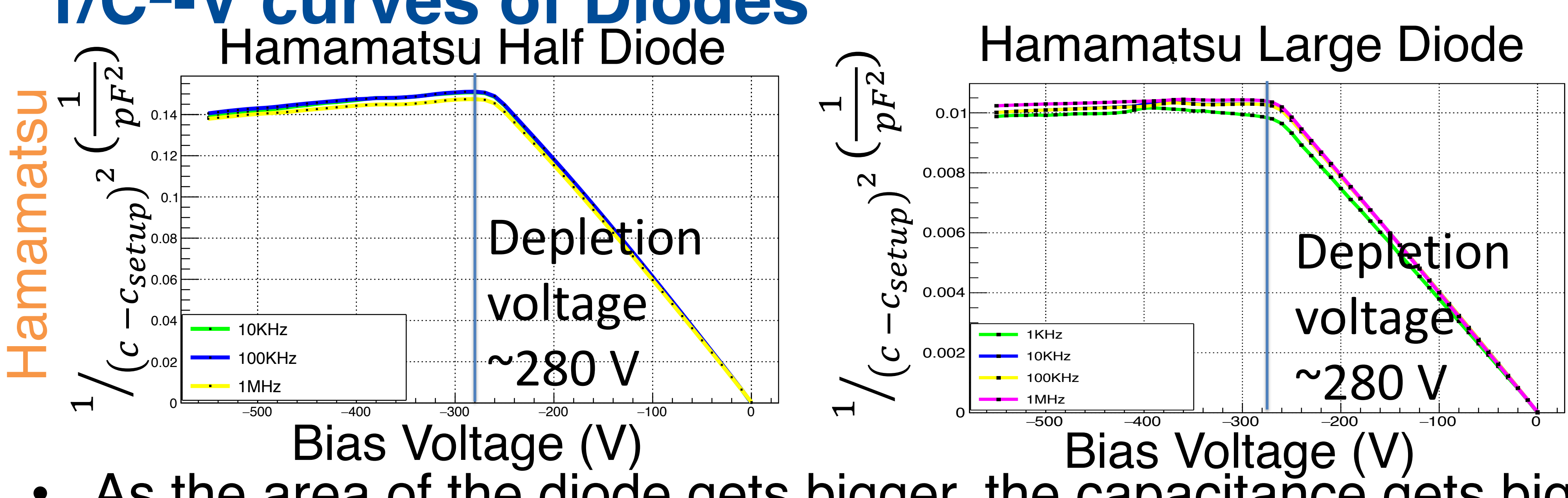

- As the area of the diode gets bigger, the capacitance gets bigger.

$C-V$ curves of MOS structures

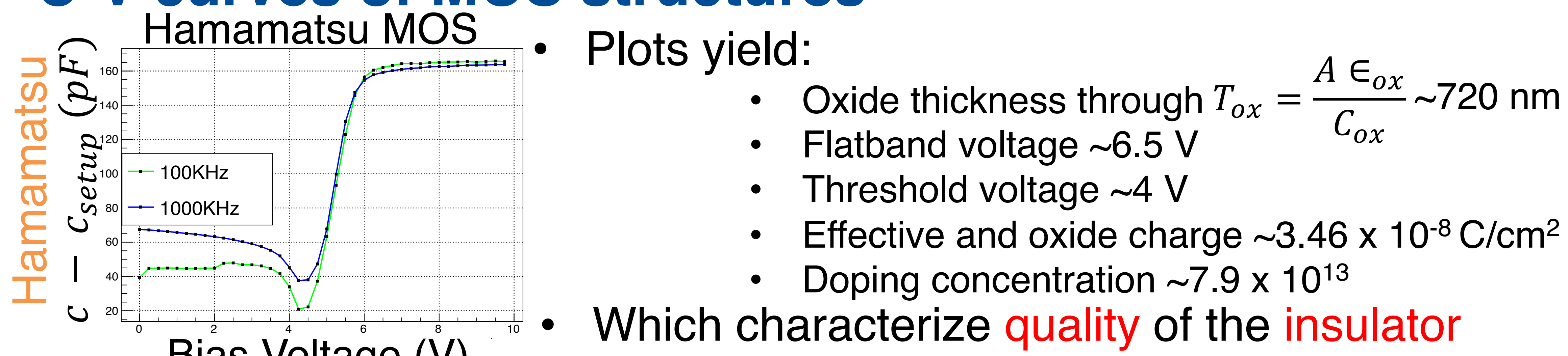

Bias Voltage $(\mathrm{V})$

- Which characterize quality of the insulator

- Hamamatsu's sensors have a thick, high-quality oxide layer.

\section{Radiation effects on MOS structures}

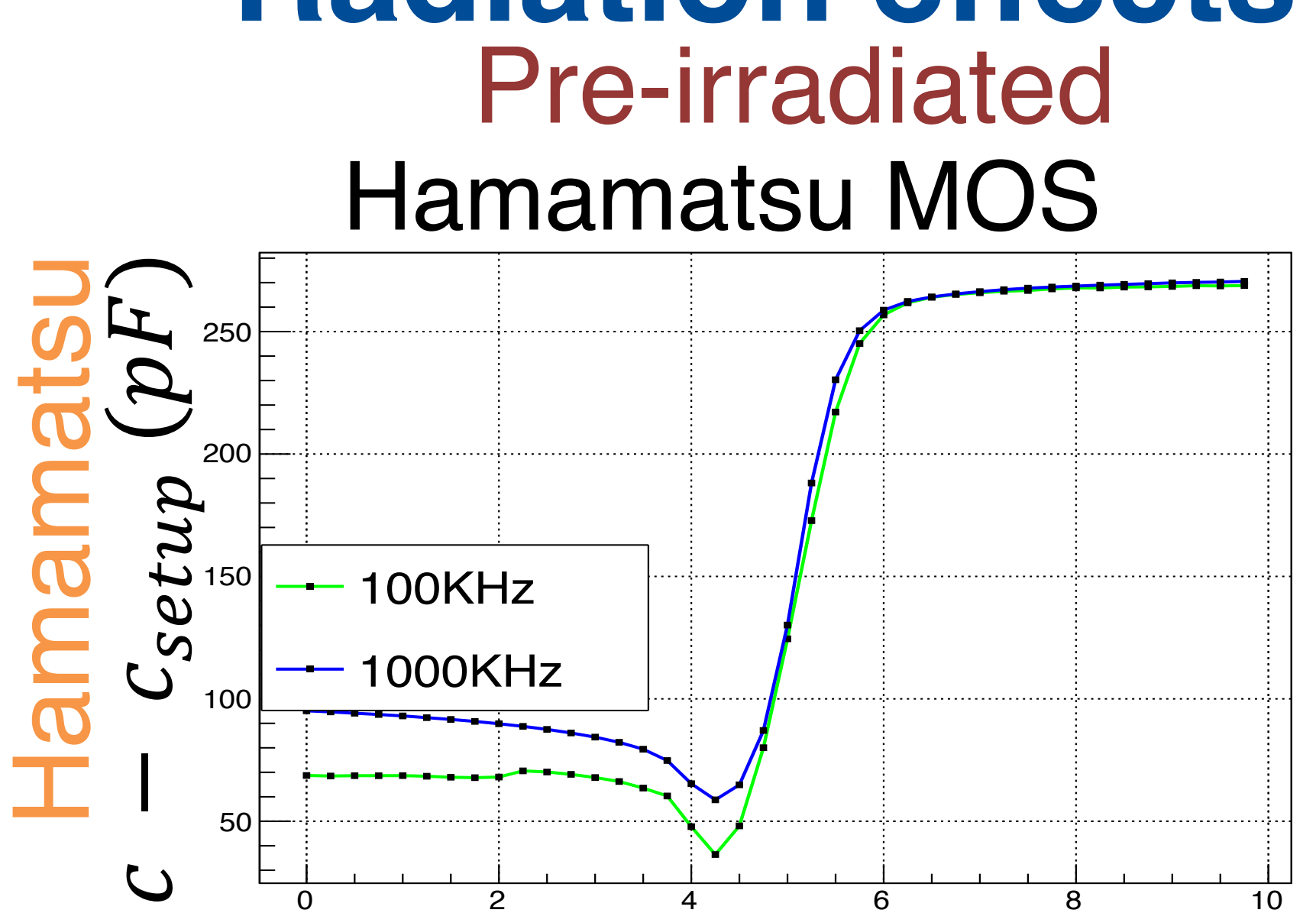

Bias Voltage (V)

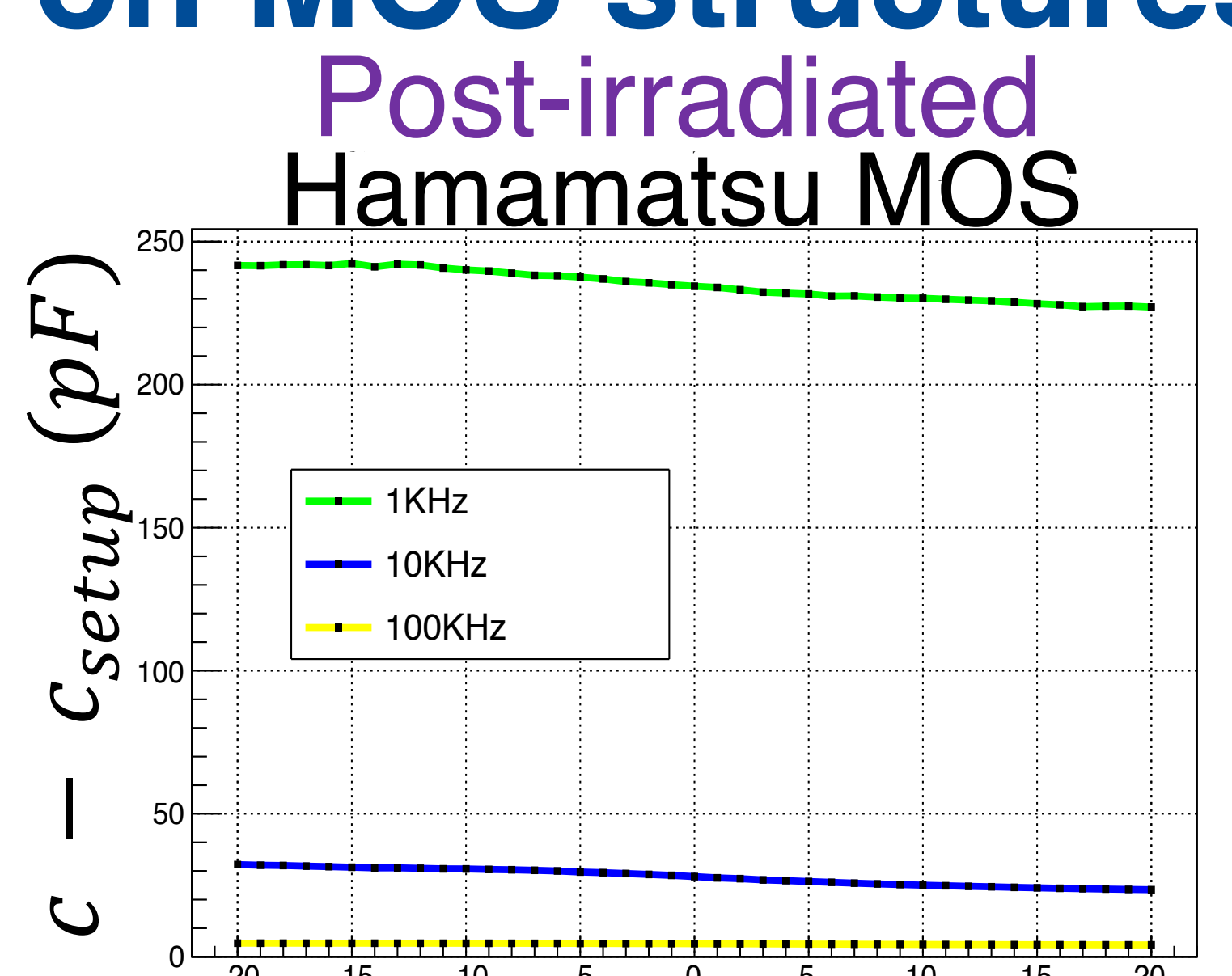

Bias Voltage (V)

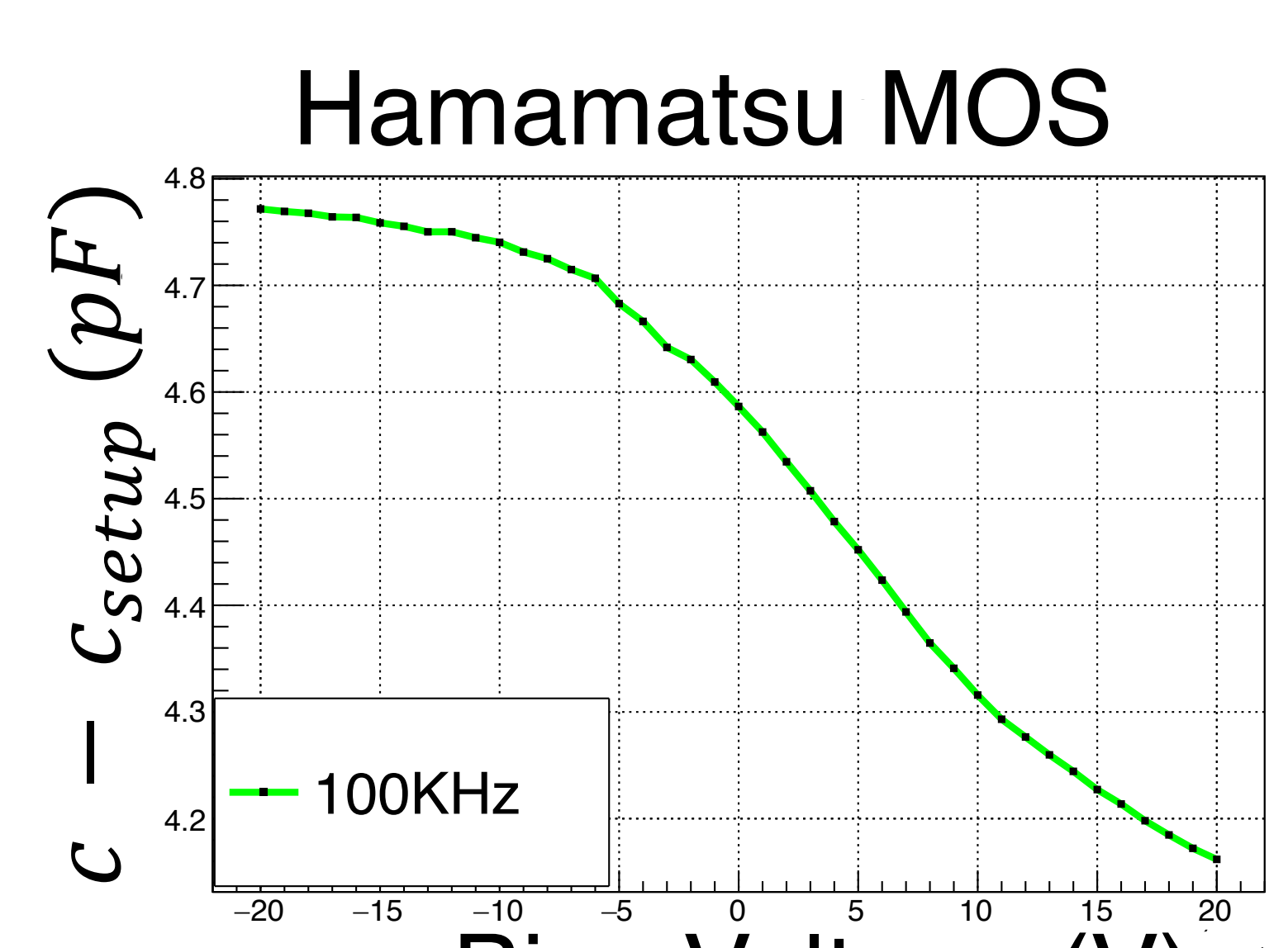

Bias Voltage (V)
- Irradiation introduces traps in oxide layer.

- Irradiated sensors have less defined curves and smaller ranges.

\section{Radiation effects on Diodes}
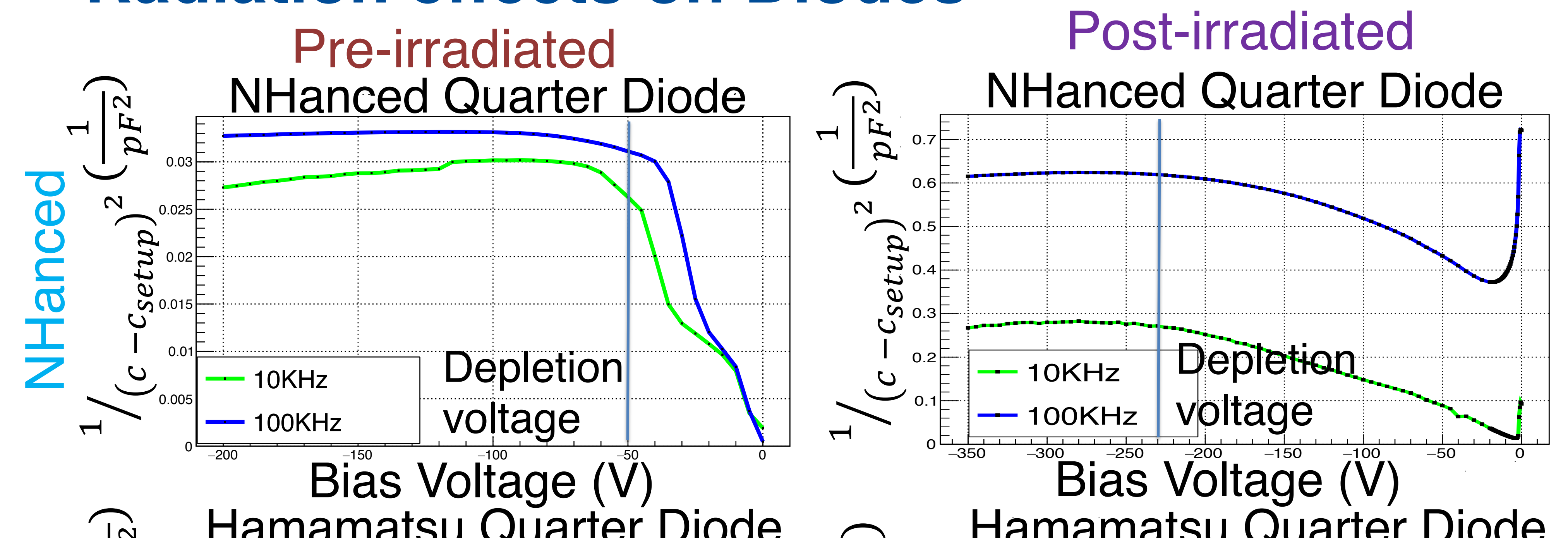

Bias Voltage (V)

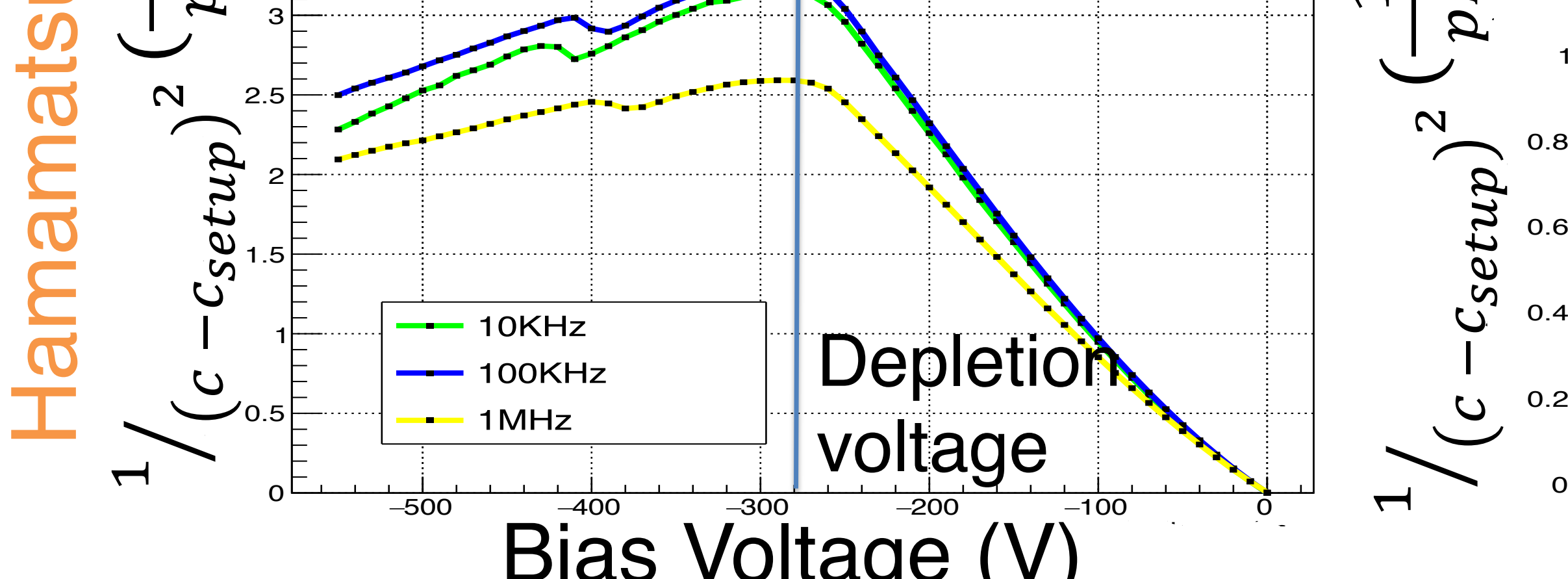

Bias Voltage $(\mathrm{V})$

- Irradiation damages the silicon structure.

- After irradiation, the shape is less defined and the depletion voltage is higher.

Conclusions

- Hamamatsu and $\mathrm{NHanched}$ both experience roughly expected radiation damage.

- Hamamatsu has better oxide quality and overall performance than Nhanced. 